\title{
FREDERICK H. HARTMANN Y LA RELACIÓN DE LAS NACIONES
}

\author{
FREDERICK H. HARTMANN AND THE RELATIONS OF NATIONS
}

\author{
Fernando D'Alessio Ipinza *
}

\section{RESUMEN}

Frederick Hartmann nació en Nueva York en 1922 y murió en el año 2015 a la edad de 93 años. Graduado de la Universidad de California en Berkeley, se incorporó a la US Navy en el programa acelerado durante la Segunda Guerra Mundial y tomó parte de nueve invasiones incluyendo la de Palau, Leyte, Linguyen, Gulf y Okinawa y recibió 7 estrellas de batalla.

En 1946 se enroló en el programa de la escuela de graduados de la Universidad de Princeton obteniendo en 1949 un PhD en Política. Fue luego profesor de la Universidad de Florida en 1948 donde fue promovido rápidamente a profesor asociado y luego a profesor principal. Hartmann comandó una división de la Reserva Naval en Gainesville, Florida y fue promovido a Capitán de Navío en 1966. Ese año aceptó una nueva posición creada en el United States Naval War College en Newport, Rhode Island, donde se desempeñó durante 20 años como profesor de Ciencias Políticas, y fue el principal asesor de nueve presidentes del Naval War College. Su prolífica producción literaria lo llevó a escribir quince libros, todos de gran reputación, entre los que destacan The Relations of Nations y The Conservation of Enemies.

Como graduado del Naval War College, me honro en intentar revivir su legado en este corto artículo para la prestigiosa Revista de la Sociedad Peruana de Derecho Internacional, en el cual trato de revisitar su libro más famoso: The Relations of Nations (1957) escrito en seis ediciones y traducido a muchos idiomas, siendo usado en escuelas de negocios internacionales, escuelas de guerra y escuela de política exterior en muchas naciones. El tema central es el que aboca al eje central de los estudios en el US Naval War College que son las relaciones internacionales y la política y estrategia en el desarrollo de las naciones.

Palabras clave: Relaciones internacionales, ley internacional, estrategia, política en las organizaciones, potencial nacional, modelo tridimensional.

\footnotetext{
* Vicealmirante AP Espada de Honor, Primer Puesto. Graduado Advanced Management Program, Harvard Business School. Doctor of Business Administration, University of Phoenix, Arizona. Engineer Degree, Master y Bachiller en Ciencias de Ingeniería Mecánica, U.S. Naval Postgraduate School, California. Master en Ciencias en Gerencia General, Salve Regina University, Newport. Licenciado en Ciencias Marítimas Escuela Naval del Perú. Doctor Honoris Causa por la Universidad Privada de Tacna. Presidente del CITME (Centro de Investigación para la Transformación y Modernidad Estratégica) Think Action Tank. Fundador de CENTRUM Católica y Director General (2000-Junio 2017). Ex Ministro de Salud del Perú.
}

Con el presente artículo, el autor formaliza su incorporación como Miembro Asociado, conforme a lo dispuesto por el Consejo Directivo de la Sociedad Peruana de Derecho Internacional, mediante Acta del 13 de diciembre de 2018. 


\section{ABSTRACT}

Frederick Hartmann was born in New York in 1922 and died in 2015 at the age of 93. He graduated from the University of California at Berkeley, he joined the US Navy in the accelerated program during World War II and took part in nine invasions including that of Palau, Leyte, Linguyen, Gulf and Okinawa and was award with 7 battle stars.

In 1946, he enrolled in the Princeton University Graduate School program, obtaining a $\mathrm{PhD}$ in Politics in 1949. He was professor at the University of Florida in 1948 where he was quickly promoted to associate professor and then to full professor. Hartmann commanded a division of the Naval Reserve in Gainesville, Florida and was promoted to Captain in 1966. That year he accepted a new position created at the United States Naval War College in Newport, Rhode Island, where he served for 20 years as Professor of Political Science and was the senior adviser to nine presidents of the Naval War College. His prolific literary production led him to write fifteen books, all of them very well known, including his most important ones: The Relations of Nations and The Conservation of Enemies.

This author as an Alum of the US Naval War College is honored to attempt to revive his legacy, in this short article, for the prestigious Journal of the Peruvian Society of International Law, in which I try to revisit his most famous book: The Relations of Nations written in 1957 with six editions and translated into many languages, being used in international business schools, war schools, and foreign policy schools in many countries. The central theme of this article is the focus of the curriculum of the US Naval War College which are the international relations and the politics and strategy in the development of nations.

Keywords: International relations, international law, strategy, policy in organizations, national potential, tridimensional model.

\section{Introducción}

Es conocido que las cinco áreas más importantes por medio de las cuales las naciones interactúan de una manera significativa son: la diplomacia, las leyes internacionales, la economía internacional, las organizaciones internacionales y el conflicto o la guerra. Teniendo como eje pivotal el de la economía internacional, ya que todo lo que ocurre en el mundo es económico. Es conocido por todos la Máxima de Carl Von Clausewitz (1832/1935) que dijo "La guerra es la continuación de la diplomacia por otros medios". Se entiende que la diplomacia es la interacción soft y la guerra es la interacción hard; y como intermediarios se encuentran las leyes internacionales, la economía internacional y las organizaciones internacionales, como son las Naciones Unidas, la OEA, entre otras. El concepto de Clausewitz $(1832 / 1935)$ era que si la diplomacia falla y todos los otros tres (las leyes internacionales, la economía internacional y las organizaciones internacionales) fallan, se deriva en una interacción hard, lo que sería la guerra o algún tipo de conflicto. Hartmann, un estudioso del tema desde 1949, escribió el libro The Relations of Nations en1957 y presentó una definición muy interesante "Las relaciones internacionales en su más amplio sentido incluyen todo tipo de interacción entre las Naciones y todos los movimientos que ocurren de personas, bienes y servicios, e ideas a través de sus fronteras y como un campo de estudio se enfoca en los procesos por los cuales los Estados ajustan sus intereses nacionales a los intereses nacionales de otros Estados", obviamente que cuando escribió esto las fronteras eran físicas, hoy en día 
tenemos que pensar de que las fronteras están abiertas por el concepto de la globalización y que tenemos una interacción también tecnológica, ello ampliaría mucho más el concepto de Hartmann, pero manteniendo la base conceptual como la misma.

La globalización es el resultado de la caída del muro de Berlín en 1989, obviamente el muro de Berlín dividía al mundo simbólicamente en dos, los que estaban con la Unión Soviética hacia el este y los que estaban con Estados Unidos hacia el oeste; el mundo vivió durante toda la etapa de la guerra fría en un mundo bipolar, por un lado controlado por Estados Unidos y por otro lado con la Unión Soviética; los países se alineaban a uno de los dos, hubieron casos excepcionales de algunos países que se alinearon con los dos, algo muy inteligente, como por ejemplo el caso de la India; se vivía en un mundo realmente estático; el Producto Bruto Interno global era muy bajo, prácticamente desde el año 1000 hasta el año 1989 el PBI global creció marginalmente, obviamente que eran etapas donde inicialmente no existían las máquinas, donde no se habían dado las revoluciones industriales, pero a pesar de todo, la producción era baja y de limitado crecimiento. En el año 1989 al caer el muro de Berlín se genera este fenómeno llamado globalización donde desaparecen las fronteras y se comienza a interactuar más agresivamente en el sentido comercial, el sentido financiero, el sentido político entre los países al desaparecer las fronteras físicas, pudiendo los países negociar con cualquier parte del mundo si es que tenían las capacidades y entrar al mercado que era el globo aquellos que podían o salir aquellos que no podían, esa fue la forma como comenzó a manejarse la economía internacional a partir del año 1989. La tecnología era muy incipiente antes de ese año, pensemos que los celulares no han existido siempre, pensemos que las computadoras que existían a comienzos de los años '70 eran esos mainframes gigantescos los cuales eran lentos y de limitada capacidad, para luego comenzar a aparecer las primeras computadoras personales en los años 1985 y en la década de los años ' 90 crece un poco más y comienza a incrementarse la capacidad de los teléfonos, la capacidad de las computadoras, la capacidad de las comunicaciones, hasta el año 2000 donde realmente se da el segundo gran cambio de la humanidad, gran cambio después del ocurrido en 1454 con la aparición de la imprenta de Johannes Gutenberg. El 2000 la economía comienza a entrar en un crecimiento exponencial y el Producto Bruto Interno se multiplica decenas de veces cada pocos años, llegamos a desarrollar una capacidad industrial realmente enorme y comienzan a aparecer los grandes polos de desarrollo tecnológico, como el de la India; reaparece un país enorme como China que despierta del letargo en que vivía; Europa que de alguna manera sigue manteniendo ese vigor industrial que tuvo desde la época de oro de la Gran Bretaña; y como mencionamos la interacción de las naciones adquiere un papel mucho más relevante donde el tema de la economía internacional juega un papel cada vez más importante.

Recordemos que en la humanidad la economía ha sido el eje central de todo, siempre la economía ha sido la que ha hecho girar a las naciones en favor a sus intereses nacionales, no existe ninguna interacción, ningún conflicto, ninguna guerra que no haya tenido un trasfondo económico y lo podemos ver hasta en la gran crisis internacional del año 2008- 2009 y esto se mantendrá porque está en el medio de estas cinco grandes áreas de la relación entre las naciones que va desde la diplomacia en un extremo, hasta el conflicto o la guerra en el otro, teniendo como eje al centro la economía internacional. Estas reflexiones nos llevan a revisitar al maestro Frederick Hartmann y su Relations of Nations

\section{La Teoría Tridimensional}

Hartmann (1957/1983) propuso esa teoría tridimensional basada en tres vértices: 1) los intereses nacionales, 2) el potencial nacional y 3) los principios cardenales, ese trípode que sin duda es el que debemos tener en consideración hoy en día cuando se piensa en negocios 
internacionales, en tratados de libre comercio, en alianzas estratégicas, en comercio tanto físico como electrónico. Es interesante esta propuesta, la cual nos lleva a analizar sobre cada uno de estos vértices (ver Figura 1).

1.- Los intereses nacionales. Son aquellos intereses supremos de un Estado, por los cuales éste haría lo posible para proteger o adquirir una relación favorable con otros Estados; los intereses nacionales no son cuantitativos, no son objetivos nacionales, son los grandes INTERESES de una nación, los cuales trata de conseguir a todo costo. La Pobreza podría ser un interés nacional, obviamente que sin indicarlo se podría entender que se trata de la eliminación o reducción de la misma. Se podría ser específico en el sentido de enunciar la eliminación de la pobreza; se puede enunciar como Bienestar de la población. Otro interés nacional puede ser la Seguridad nacional o la Protección de nuestros valores. Son enunciados macro, los cuales se pueden convertir en Políticas de Estado, para más adelante se puedan derivar en los objetivos nacionales, los cuales sí son cuantitativos, medibles, y comparables. Este primer vértice de intereses nacionales no está grabado en piedra, de alguna manera son la base de la política exterior de los países y lógicamente cuando el entorno cambia se pueden ajustar a la nueva realidad. El entorno cambia muchas veces radicalmente como en la crisis financiera del año 2009 o en el presente año por el Covid-19 y seguirá siendo impredecible y volátil, por eso las naciones deben ser muy cuidadosas al enunciar sus intereses nacionales, pero hay que anunciarlos sin duda alguna. Un país que no tenga intereses nacionales, aunque no deben ser muchos, sería un país sin brújula y a la deriva. Se puede hablar de unos 4, 5 o 6 grandes intereses nacionales, los cuales son los que agrupan las mayores necesidades o los mayores deseos de un país para conseguirlos, como el interés porque su población esté bien alimentada, con buena salud, buena educación, buenos ingresos económicos que lo llevarán a ser un país desarrollado finalmente. Acerca de estos intereses nacionales Hartmann (1957/1983) indicó que hay dos grandes tipos de intereses nacionales: (a) los comunes y (b) los opuestos, en relación con los intereses nacionales de otros países. (a) Si dos países tienen intereses nacionales comunes, podrían llegar fácilmente a alianzas, acuerdos o convenios para que los dos se favorezcan de una manera muy positiva y ellos hay que buscarlos; (b) el otro gran interés, como su nombre indica, es el interés opuesto, el cual no ayuda a hacer ningún tipo de alianza y es mejor mantener esa relación en el limbo, el tratar de hacer una alianza o un convenio con un país con el cual tenemos un interés opuesto es una receta al fracaso, porque no se va a poder conseguir y eso se debe tener muy claro.

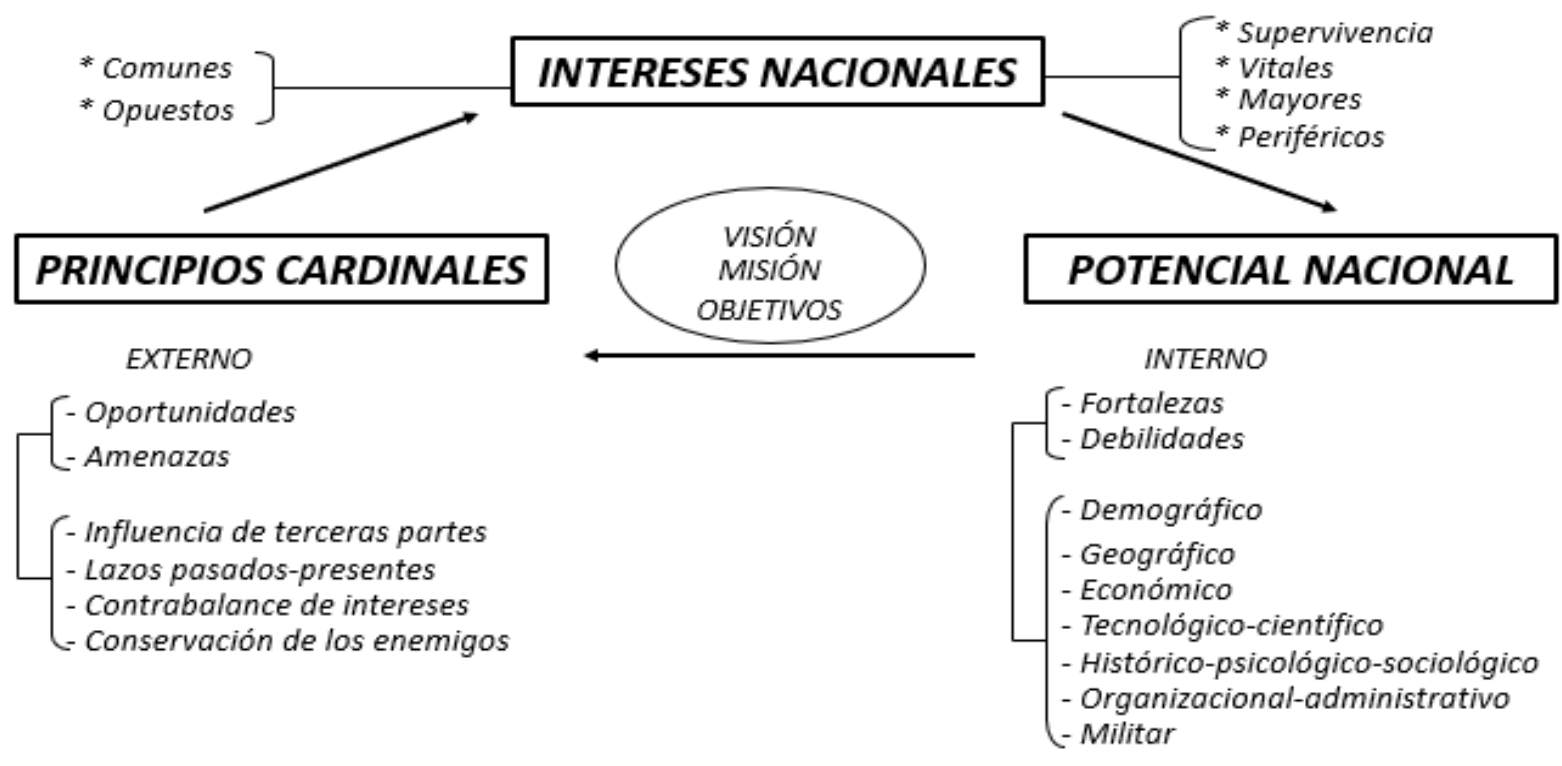


Figura 1. Teoría tridimensional de las relaciones entre países. Adaptado del original y tomado de El Proceso Estratégico: Un enfoque de gerencia, F. D’Alessio (2013).

Hartmann (1957/1983) califica los intereses nacionales en cuatro niveles de intensidad en sus relaciones con otras naciones, sean estos comunes u opuestos: (1) El primer nivel de intensidad es el de Supervivencia, es un interés que si fuera opuesto pondría en serio peligro la vida del Estado o si fuera común, el interés los ayudaría sustancialmente a desarrollarse y crecer; sin lugar a dudas, en ambos casos el interés es de supervivencia. Como ejemplo de Supervivencia opuesta, es el que tuvieron la Unión Soviética con los Estados Unidos en la época de la Guerra Fría; el primero, si hubiera disparado misiles intercontinentales podría haber hecho desaparecer al otro y posiblemente, por la distancia entre ellos, si ambos disparasen al mismo tiempo, los dos desaparecerían, o sea el interés de supervivencia opuesto es un interés de vida o muerte. (2) El segundo nivel de los intereses nacionales son los intereses Vitales, son aquellos, como su nombre indica, afectan la vida de una Nación y la podrían poner en peligro, favoreciendo o impidiendo su desarrollo. (3) El tercer nivel son los intereses Mayores, que pueden afectar o favorecer la situación de un país, es decir, actúan en favor o en contra. (4) El cuarto nivel Periféricos, son molestos y de alguna manera perturban al país en alcanzar sus intereses. Estos cuatro niveles tienen las dos posiciones, pueden ser comunes u opuestos, en cada nivel de intensidad tendría que colocarse con qué países puedo tener algún interés opuesto y con cuál se podrían tener intereses comunes. Los intereses opuestos van a perjudicar el alcanzar el interés nacional y los comunes, favorecen a alcanzarlos. Un ejemplo real podemos verlo con la matriz de Intereses Nacionales de los Estados Unidos en la Tabla 1, en el cual se muestra cómo Estado Unidos mantiene los mismos cuatro intereses nacionales hace mucho tiempo y en dicha matriz asigna los países con los que tiene intereses comunes con un asterisco (*) y con los cuales tiene intereses opuestos dos asteriscos (**), ahora lo importante para Estados Unidos es que esos cuatro intereses nacionales han permanecido y lógicamente con los años van actualizando los países que la conforman, de acuerdo a las evoluciones del entorno y sus efectos geo-estratégicos.

Tabla 1

Matriz Interés Nacional de Estados Unidos de América

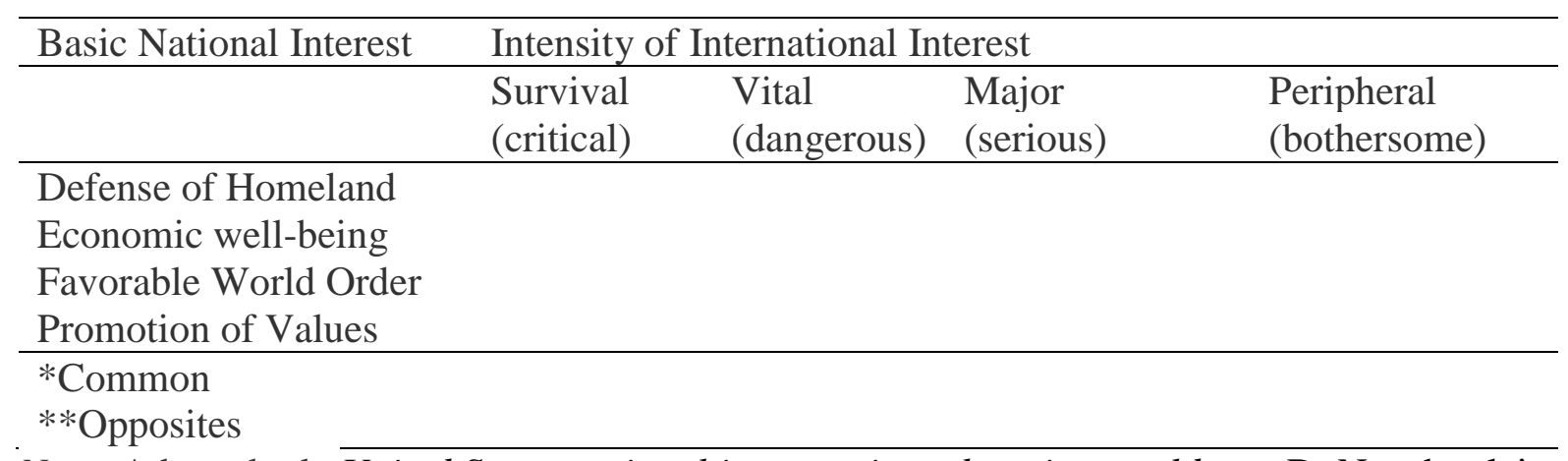

Nota. Adaptado de $\overline{\text { United States national interests in a changing world, por D. Nuechterlein, }}$ 1973.

2.- El potencial nacional. Este vértice debe ser analizado en función a la seguridad del país y sus patrones del poder. La seguridad nacional se basa en tres posibilidades en función a cómo los Estados entran en arreglos de seguridad según las estructuras que pueden manejar el 
poder. (1) En primer lugar, la seguridad es una condición relativa de cada país, es más deseada por los Estados que la paz per se y tiene diferentes connotaciones en función a cada país; Hartmann (1957/1983) definió la Seguridad como la suma total de los intereses nacionales vitales, porque los intereses vitales de una nación son aquellos que están dispuestos a utilizar o no la fuerza. (2) En segundo lugar, cuando las naciones se agrupan hacen acuerdos o alianzas de formas diferentes, las cuales van desde el unilateralismo, que significa ir solo contra el mundo y no tener ningún tipo de alianza, hasta las alianzas colectivas que se buscan para tener un balance del proceso del poder. (3) En tercer lugar, la seguridad colectiva es agruparse con otros países ya sea regional o globalmente, se puede tener el caso de conseguir una federación voluntaria mundial, algo así como el papel que juega las Naciones Unidas en este momento.

Estos patrones de la seguridad y del poder se basan en el Potencial con que cuenta cada país, este potencial se mide en función a siete grandes potenciales factores que poseen como son: (a) demográfico, (b) geográfico, (c) económico, (d) tecnológico-científico, (e) históricopsicológico-sociológico, (f) organizacional-administrativo, y (g) militar. Analizando cada uno de estos factores se pueden obtener las fortalezas y debilidades del país, lo que hace visualizar qué potencial tiene; recordemos que el potencial es algo estático, es algo que se tiene, pero todavía no se ha puesto en marcha, es como si se hiciera una equivalencia entre la energía potencial que tienen los cuerpos en función a su posición y lugar en el planeta, con relación a lo cinético que sería el que tienen con relación a sus movimientos, entonces se debe actuar sobre el potencial para en base a ello desarrollar favorablemente las ventajas competitivas del país. Pongamos como ejemplo nuevamente a los Estados Unidos de Norteamérica, la gran ventaja competitiva que tiene es el factor militar, porque todas las industrias que desarrollan su poder bélico se producen en el mismo país, no tienen que importar nada a nadie, eso crea industrias colaterales, genera empleo, le produce riqueza al país y realmente, para Estados Unidos tener un poder bélico es incrementar su Producto Bruto Interno monumentalmente; en cambio, para los países en vía de desarrollo esto no es así, lo militar es un gasto, porque hay que importar casi todo el material que se usa y eso resulta en mermas presupuestales, la capacidad de usar el presupuesto un país debe ser mayormente para funciones sociales u otras que tienen un mayor rédito. En ese sentido lo militar para un país es una debilidad y para el otro, es una fortaleza, y así es como podemos analizar cada uno de los siete factores.

3.- Los principios cardinales. Este es un concepto sumamente interesante que plantea Hartmann (1957/1983), porque menciona que los Estados cuando interactúan entre sí tienen cuatro formas de hacerlo: (1) el primer principio es la Influencia de terceras partes, ya que indica que ninguna interacción entre dos partes es puramente bilateral y siempre habrán influencias de terceras o cuartas partes, pensar que algo puede ser bilateral es realmente una ingenuidad, en el caso de Malvinas, los argentinos creyeron que era algo entre ellos y los británicos, ya que si hubiera sido así de repente hubieran ganado, pero otros observaban y apoyaron abiertamente a los británicos inclinando a su favor el resultado del conflicto; (2) como segundo principio tenemos los Lazos pasados y presentes, lo que significa que el pasado va a proyectarse al presente, no se puede borrar ni puede desaparecer, es otra ingenuidad pensar que el pasado ya desapareció; (3) como tercer principio está el Contrabalance de intereses, es claro que para hacer un acuerdo o una alianza se debe tener un interés común, es otra ingenuidad el pensar hacer una alianza con quien se tiene un interés opuesto; por último, (4) está la Conservación de los enemigos, que es algo muy interesante e importante y que generó un libro completo escrito por Hartmann (1957/1983), lo que indica es que no hay que ganar ni perder enemigos, hay que conservarlos y mantenerlos, eso es interesante porque lógicamente el tener enemigos genera estar en alerta, estar trabajando y no descuidarse, son casos muy raros en el 
mundo donde algún Estado no ha tenido nunca enemigos y este principio cardinal no contaría, casos excepcionales de países como Suiza y Costa Rica. Ahora, sí hubo casos de ganar o perder enemigos, los cuales tuvieron resultados funestos para los que lo hicieron.

\section{Reflexiones}

La contribución de Hartmann en la Relación de las Naciones es sumamente importante, los países deberían intentar seguir el formato de su Teoría Tridimensional escribiendo cada uno de sus vértices para tener muy clara la necesaria política exterior del país y las políticas de Estado que desarrollarán para alcanzar el bienestar y seguridad de sus poblaciones. El primer paso sería enunciar los intereses nacionales, que como se dijo no deben ser muchos, sólo 4, 5 o 6, no más y deben ser enunciados en forma precisa, como se vio en la matriz presentada en la Tabla 1 correspondiente a los Estados Unidos, se menciona cuatro intereses nacionales: Bienestar de su población (Economic well-being), Defensa del territorio (Defense of homeland), Orden favorable mundial (Favorable world order) y Promoción de valores occidentales (Promotion of values), se borró la palabra occidentales, pero lo que ellos querían decir era que serían los que iban a dirigir la preservación de estos valores en el mundo. Se debe establecer la Matriz de Intereses Nacionales, en la cual se tienen los intereses nacionales por un lado y por el otro, los cuatro niveles de intensidad de los intereses que son Supervivencia, Vital, Mayor y Periférico; dentro de la matriz se colocan aquellos países con los cuales se tiene un interés común y con los que se tiene un interés opuesto en cada uno de los casilleros, los cuatro niveles de intensidad versus los intereses nacionales, en el caso de la matriz de Estados Unidos serían 16 casilleros, donde se pondrían a los países con un asterisco $\left(^{*}\right)$ con aquellos países con los cuales se tiene un interés común y con dos asteriscos $(* *)$ con aquellos que se tiene un interés opuesto. Co eso se comienza una Política Exterior muy elaborada, tratando de afianzar lazos con aquellos países que se tenga un interés común y tratando de neutralizar, o dejar en el limbo, con los cuales se tenga un interés opuesto. El segundo paso es evaluar el Potencial Nacional, como bien se dijo, el potencial es aquello que un país puede hacer, no significa que eso ya lo tiene, sino es el potencial nacional que debe convertirlo en ventajas competitivas; basadas en los siete grandes factores que constituyen el Potencial Nacional: demográfico, geográfico, económico, tecnológico-científico, histórico-psicológicosociológico, organizacional-administrativo, y militar; para convertir sus ventajas comparativas en ventajas competitivas, con las cuales se podrá salir a los mercados internacionales y mejorar la balanza de pagos, satisfacer la demanda interna, y hacer crecer sostenidamente el Producto Bruto Interno, que si bien es una medida bastante relativa, le da una idea a los otros países de cómo está nuestro país en relación a ellos, ajustado esa medida a una Paridad de compra ajustada (Purchasing Power Parity). El tercer paso, los Principios Cardinales, que es lo más interesante, lo más analítico, y lo más estratégico de cómo manejar estas interacciones con los países y es el punto capital de la Teoría Tridimensional de la Naciones de Hartmann, con quiénes puedo desarrollar acuerdos o no, basado también en el pensamiento de que en todo acuerdo van a existir influencias de terceras partes que podrían bloquearla, contaminarla o impedirla, o aquellos que podrían favorecerla, ayudarla a ser una alianza, mejorarla, como el caso de la Alianza del Pacífico, que considero está siendo poco usada, a pesar de ser de lo más importante que ha hecho el Perú en términos de relaciones internacionales, se ha dicho que manejar bien la Alianza del Pacífico pondría a los cuatro países miembros (Perú, Chile, Colombia y México) convertirlos en el octavo lugar de las economías mundiales, desafortunadamente el Perú no es miembro la OCDE como sí ya lo son los otros tres países, y eso realmente marca una diferencia que debe ser superada rápidamente; en referencia a los 
lazos pasados y presentes no se debe pensar que el pasado se borró para siempre o que ya no existe, no, ahí está latente, y se proyecta al presente y el futuro; el contrabalance de intereses, a no intentar hacer una alianza con quien se tenga intereses opuestos, eso sería una ingenuidad y se perdería tiempo, dinero y esfuerzo; por último, la conservación del enemigo, aunque parece una paradoja o una sin razón, porque muchos dirían que lo mejor es no tener enemigos, sería lo ideal y viviríamos en un mundo de paz eterna, pero la realidad es que los enemigos siempre están presentes, lo que para las empresas se llaman competidores, para en la política exterior se llaman enemigos, recordar la máxima de Carl von Clausewitz "la guerra es la continuación de la diplomacia por otros medios". Creo que si se dan estos pasos muy bien estudiados, el Perú tendría un esquema con el cual podría manejar muchas cosas hacia el exterior y hacia el interior, reflexionemos sobre estos aspectos con los que se podría mejorar muchísimo.

\section{Referencias}

Clausewitz, K. Von (1832/1935). Vom Kriege [On war]. Leipzig, Alemania: Insel Verlag.

D’Alessio, F. (2013). El Proceso Estratégico: Un enfoque de gerencia. México: Pearson.

Hartmann, F. H. (1957/1983). The relations of nations. Nueva York: Macmillan.

Nuechterlein, D. (1973). United States national interests in a changing world. Lexington, KY: University Press of Kentucky. 